\title{
Tumor detection with 5-aminolevulinic acid fluorescence and Gd-DTPA-enhanced intraoperative MRI at the border of contrast-enhancing lesions: a prospective study based on histopathological assessment
}

\author{
Jan Coburger, M.D., ${ }^{1}$ Jens Engelke, M.D., ${ }^{1}$ Angelika Scheuerle, M.D., ${ }^{2}$ \\ Dietmar R. Thal, M.D., ${ }^{2}$ Michal Hlavac, M.D., ${ }^{1}$ Christian Rainer Wirtz, M.D., Ph.D., ${ }^{1}$ \\ and Ralph König, M.D., Ph.D. ${ }^{1}$ \\ ${ }^{1}$ Department of Neurosurgery, and ${ }^{2}$ Section of Neuropathology, University of Ulm, Günzburg, Germany
}

\begin{abstract}
Object. High-grade gliomas (HGGs) and metastasis (MET) are the most common intracranial lesions in neurosurgical routine. Both of them show an invasive growth pattern extending into neural tissue beyond the margins of contrast enhancement on MRI. These "undetected" areas might be the origin of early tumor recurrence. The aim of the present study was to evaluate whether 5-aminolevulinic acid (5-ALA) fluorescence provides an additional benefit in detection of invasive tumor compared with intraoperative MRI (iMRI).

Methods. The authors prospectively enrolled 45 patients harboring contrast-enhancing lesions, in whom grosstotal resection was intended. All patients had surgery in which iMRI and 5-ALA-guided resection were used following a specific protocol. First, a typical white light tumor resection was performed. Then, spatial location of residual fluorescence was marked. After that, an iMRI was performed and residual uptake of contrast was marked. Navigated biopsy samples were taken from all marked areas and from additional sites according to the surgeon's judgment. Cross tables and receiver operating characteristic curves were calculated, assessing performance of the imaging methods for tumor detection alone and for combined detection of infiltration zone and solid tumor (pathological tissue). Also, correlations of histopathological findings with imaging results were tested using Spearman rho.

Results. Thirty-four patients with HGGs and 11 with METs were enrolled. Three patients harboring a MET showed no 5-ALA enhancement and were excluded; 127 histopathological samples were harvested in the remaining patients. In HGG, sensitivity for tumor detection was significantly higher $(\mathrm{p}<0.001)$ in 5-ALA $(0.85)$ than in iMRI (0.41). Specificity was significantly lower $(\mathrm{p}<0.001)$ in 5-ALA $(0.43)$ than in iMRI $(0.70)$. For detection of pathological tissue, 5-ALA significantly exceeded iMRI in specificity ( $0.80 \mathrm{vs} 0.60)$ and sensitivity (0.91 vs 0.66$)(\mathrm{p}$ $<0.001$ ). Imaging results of iMRI and 5-ALA did not correlate significantly; only 5-ALA showed a significant correlation with final histopathological diagnosis of the specimen and with typical histopathological features of HGGs. In METs, sensitivity and specificity for tumor detection were equal in 5-ALA and iMRI. Both techniques showed high values for sensitivity $(0.75)$ and specificity $(0.80)$. The odds ratio for detection of tumor tissue was 12 for both techniques. Concerning pathological tissue, no statistically significant difference was found either. Imaging results of iMRI and 5-ALA correlated significantly $(\mathrm{p}<0.022)$, as with final histopathological diagnosis in METs.

Conclusions. In METs, due to the rate of nonenhancing lesions, the authors found no additional benefit of 5-ALA compared with iMRI. In HGG, imaging results of 5-ALA and iMRI are significantly different at the border zone; 5-ALA has a higher sensitivity and a lower specificity for tumor detection than Gd-DTPA-enhanced iMRI. For detection of infiltrating tumor at the border of the resection cavity, 5-ALA is superior to Gd-DTPA-enhanced iMRI concerning both sensitivity and specificity. Thus, use of 5-ALA in addition to iMRI might be beneficial to maximize extent of resection. Clinical synergistic effects will be evaluated in a prospective randomized trial. (http://thejns.org/doi/abs/10.3171/2013.11.FOCUS13463)
\end{abstract}

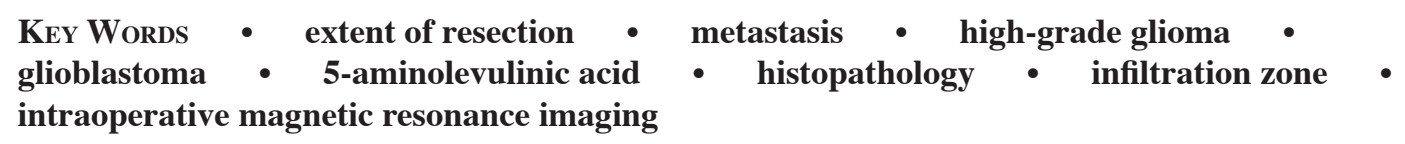

\footnotetext{
Abbreviations used in this paper: AUC = area under curve; 5-ALA = 5-aminolevulinic acid; EOR = extent of resection; GBM = glioblastoma multiforme; HGG = high-grade glioma; iMRI = intraoperative MRI; MET = metastasis; MPRAGE = magnetizationprepared rapid acquisition gradient echo; NPV = negative predictive value; $\mathrm{OR}=$ odds ratio; $\mathrm{PPV}=$ positive predictive value; $\mathrm{ROC}=$ receiver operating characteristic.
}

EVERAL studies have shown that the extent of resec-
tion (EOR) correlates with increased survival in pa-
tients with high-grade gliomas (HGGs).,5,11,12,14,16,20,32
For 5-aminolevulinic acid (5-ALA) fluorescence and for
low-field intraoperative MRI (iMRI), Class I evidence
exists to increase EOR. ${ }^{22,24}$
The outline of contrast enhancement in Gd-DPTA- 


\section{J. Coburger et al.}

enhanced MRI is the most widely accepted target area of HGG and metastasis (MET) surgery. Most large trials, including the above-mentioned publications, are based on the EOR calculated on pre- and postoperative contrast enhancement. ${ }^{14,26}$ However, a sharp delineation of the tumor as indicated by contrast enhancement cannot be presumed, due to the invasive nature of an HGG. A comparison of imaging results and postmortem histopathological findings showed that $60 \%-100 \%$ of proliferating tumor cells can be found from 6 to $14 \mathrm{~mm}$ beyond the margins of contrast enhancement in HGGs. ${ }^{30}$ Similar findings were published for brain METs. Therefore a "safety margin" of $5 \mathrm{~mm}$ beyond the contrast-enhancing lesion or a "supramaximal" resection are suggested by some authors.7,31

These infiltrating areas are clinically significant; they might be the origin of early recurrence both for HGGs and METs. Therefore the border zone of contrast-enhancing brain tumors is of special relevance. For the first time, the present study evaluates the sensitivity and specificity of 5-ALA- and Gd-DTPA-enhanced iMRI to detect tumor in that border zone of HGGs and METs. Because 5-ALA is based on a cellular accumulation of protoporphyrin IX, it might depict tumor invasion differently from Gd-DTPA enhancement, being based on blood-brain barrier rupture. Yet, data published so far referring to HGG postulate a close correlation of preoperative MRI and intraoperative 5-ALA fluorescence. ${ }^{17,24}$ However, a direct comparison of preoperative imaging and intraoperative 5-ALA is hampered due to brain shift during tumor resection. Therefore a direct spatial correlation of the Gd-DPTA enhancement with intraoperative fluorescence is only possible with an intraoperative update of imaging in which iMRI is used. For METs, to our knowledge, information about correlation of Gd-DTPA-enhanced MRI and 5-ALA fluorescence is still lacking.

Our aim was to provide a histopathologically based correlation of tumor delineation at the border zone of GdDTPA-enhanced iMRI and 5-ALA in contrast-enhancing lesions.

\section{Methods}

\section{Study Design}

We used a prospective nonrandomized study design and included patients harboring a contrast-enhancing intraaxial lesion who were eligible for the study, after giving informed consent, from July 2012 to July 2013. Approval was received from the local ethics committee (Ethik Kommission Universität Ulm; approval no. 172/12).

Primary inclusion criteria were a planned gross-total resection, no contraindication for 5-ALA or iMRI, and informed consent by the patient. Exclusion criteria were an age younger than 18 or older than 75 years.

\section{Study Protocol}

A Gd-DTPA-enhanced MRI study was obtained in all patients at a maximum of 1 week prior to surgery. The 5-ALA was administered orally 4 hours before surgery in a common dose of $20 \mathrm{mg} / \mathrm{kg}$ body weight. Patients underwent operation in a BrainSuite iMRI Miyabi equipped with a 1.5-T Magnetom Espree scanner (Siemens).
Patient registration to the neuronavigation system was performed using automatic registration tools (BrainLab) on the basis of a short preoperative T1 magnetization-prepared rapid acquisition gradient echo (MPRAGE) scan without contrast after fixation of the head in the Noras head coil. First, a typical white light resection was performed under neuronavigation guidance, until the surgeon judged that removal of the tumor was complete. After hemostasis was obtained, illumination of the microscope was switched to 405-nm fluorescence and resection control was performed using 5-ALA. The exact spatial localization of fluorescent tissue was marked and stored in the neuronavigation system (BrainLab).

Subsequently a session of contrast-enhanced iMRI (T1 MPRAGE) was conducted. Residual tumor in contrast-enhanced iMRI was marked and volumetric assessment performed by applying the neuronavigation software. Using the navigation microscope, we then consecutively displayed all previously marked 5-ALA-positive points and compared the results with findings on iMRI. In return all Gd-DTPA-positive areas were scanned using 405-nm fluorescence if not detected before.

Depiction of residual tumor by 5-ALA and iMRI was rated by the surgeon as follows: 5-ALA fluorescence was graded as "negative," "intermediate," or "positive." "Positive" fluorescence was defined as typically bright, glowing, magenta fluorescence as recommended for resection in the 5-ALA trial published by Stummer et al. ${ }^{24}$ "Intermediate" fluorescence was defined as a low-intensity pinkish fluorescence, also described as "vague" fluorescence in the above-mentioned trial. "Negative" was defined as bluish or absent fluorescence.

Findings in contrast-enhanced iMRI were graded as "negative," "intermediate," and "positive." We described the image result as "intermediate" if it differed significantly from "normal" tissue or edema but also differed from the areas we would classify as positive for tumor (for example, a dull contrast enhancement in a glioblastoma).

Tissue samples of tumor-positive sites found on iMRI or 5-ALA studies were harvested with navigated biopsy procedures according to the previously acquired spatial objects. Samples from negative areas in both imaging techniques were taken at the discretion of surgeon.

\section{Microscopy Apparatus}

A Zeiss Pentero 600 with integrated head-up display was used for visualization of neuronavigation and a Blue 400 filter was used to perform 405-nm fluorescence.

\section{Neuronavigation/MRI Unit}

We performed all surgeries in a dedicated 1.5-T iMRI (Magnetom Espree, Siemens Healthcare) environment with integrated data management and neuronavigation (Brainsuite, BrainLab). The iPlan 3.0 software (BrainLab) was used.

\section{Histopathological Assessment}

Samples for histopathological assessment were categorized by the neurosurgeon according to the imaging results of iMRI and 5-ALA and forwarded to the neuro- 


\section{Histopathological assessment at border zone with 5-ALA and iMRI}

pathology department as separate samples. The neuropathologists were blinded to the clinical categorization of the samples. Two neuropathologists observed the samples separately; in case of disagreement, they discussed the sample and came to a consensus diagnosis.

All samples were fixed in $10 \%$ buffered formalin and embedded in paraffin. Human glioblastomas multiforme (GBMs) were classified neuropathologically according to the WHO CNS tumor classification. ${ }^{13}$ For this purpose paraffin sections were stained with $\mathrm{H} \& \mathrm{E}$. Immunohistochemical investigation for GBMs was performed with antibodies raised against glial fibrillary acidic protein (polyclonal rabbit, 1:1000, DAKO), microtubule-associated protein (HM-2, 1:500, heat pretreatment, Sigma-Aldrich), and the Ki 67 epitope (MIB-1, 1:100, heat pretreatment, DAKO). Metastatic brain lesions of carcinomas were stained for cytokeratin (MNF116) and the Ki 67 antigen. Pathologically, "solid tumor" was defined as tumor tissue exhibiting a pattern fulfilling the WHO diagnostic criteria of the given tumor entity with less than $20 \%$ nontumor tissue at the $\mathrm{H} \& \mathrm{E}$ level. "Tumor infiltration zone" samples contained less than $20 \%$ tumor cells, but tumor cells and tumor proliferation were detectable in $\mathrm{H} \& \mathrm{E}$ and anti-Ki 67-stained samples. "Tumor-free tissue" referred to samples in which no tumor cells could be identified at the $\mathrm{H}$ \& E level and no increased levels of proliferation were detectable with anti-Ki 67.

\section{Statistical Assessment}

The correlation of imaging methods was tested using Spearman rho. For histopathological evaluation of imaging methods we used descriptive statistics and calculated sensitivity (true-positive rate), specificity (true-negative rate), positive predictive value (PPV) and negative predictive value (NPV), and odds ratio (OR) to detect solid tumor.

After dichotomizing the data we used the McNemar test to assess whether there is a difference of distribution to detect tumor-positive areas (sensitivity) and tumornegative areas not exhibiting features of the main tumor (specificity) between iMRI and 5-ALA. Negative areas were defined as areas not exhibiting all features of the main tumor (infiltration zone) and were most likely to be tumor-free tissue.

We calculated the receiver operating characteristics (ROCs) and the area under curve (AUC). Additionally, all parameters were calculated for detecting pathological tissue in general. We defined pathological tissue as tumor area with features of the main tumor and areas not exhibiting all features of the main tumor (infiltration zone). Negative areas were defined as most likely to be tumorfree tissue in this calculation.

Statistical calculations were performed using SPSS version 15.0 (Lead Technologies, Inc.).

\section{Results}

\section{Patient Population}

Forty-five patients (18 female and 27 male) were enrolled from July 2012 to July 2013. Three of 11 patients harboring a MET had to be excluded from the study be- cause the tumor tissue appeared to be fluorescence negative intraoperatively. The final study group consisted of 34 patients with HGGs and 8 with METs.

Final histopathological assessment confirmed WHO Grade IV GBM in those 34 patients with HGG. Without exception, fluorescence-negative METs turned out to be from adenocarcinoma. Histopathological examination revealed adenocarcinoma in 5 and squamous cell carcinoma in 3 of the remaining 5-ALA-positive METs. A detailed listing of patient characteristics is found in Table 1. After white light resection, 127 histopathological samples were harvested; 114 were taken from HGGs and 13 from METs.

\section{Comparison of Histopathological Results: HGGs}

Distribution of test results is distinctly different in 5-ALA and iMRI (Table 2). In iMRI the number of specimens that tested positive for tumor or infiltration zone is shifted to the left side of the table, representing an "under detection," whereas in 5-ALA the opposite is the case, and a slight "over detection" can be noticed. Using 5-ALA and iMRI we have found a high rate of falsepositive detection of infiltration zone as tumor.

Calculations of test performance for tumor detection in HGG are found in Table 3. The false-negative rate was higher in iMRI (0.47) in comparison with 5-ALA (0.33). The false-positive rate was comparable in both techniques (0.33 in iMRI vs 0.31 in 5-ALA), as was the PPV, which was 0.67 in iMRI and 0.69 in 5-ALA. The NPV was higher in iMRI (0.70) in comparison with 5-ALA (0.43). However, the share of "undetected" sites representing areas with histopathological evidence of solid tumor in a specimen rated negative or intermediate on imaging was distinctly higher in iMRI $(\mathrm{n}=40,59 \%)$ compared with 5-ALA ( $\mathrm{n}=10$, $15 \%$ ). The OR for solid tumor in an area labeled positive on intraoperative imaging was 1.6 for iMRI and 4.5 with 5-ALA. This finding is reflected in the calculated sensitiv-

TABLE 1: Characteristics of 42 patients with contrast-enhancing intracranial lesions*

\begin{tabular}{lc}
\hline \multicolumn{1}{c}{ Characteristic } & Value \\
\hline mean age in yrs (range) & $59(32-82)$ \\
female ratio & $40 \%$ \\
location of tumor & \\
frontal & $41 \%$ \\
temporal & $31 \%$ \\
parietal & $14 \%$ \\
occipital & $12 \%$ \\
cerebellar & $3 \%$ \\
It-sided lesion & $42 \%$ \\
recurrent disease & $17 \%$ \\
Dx (no. of patients) & \\
GBM & 34 \\
MET & \\
adenocarcinoma & 5 \\
squamous cell carcinoma & 3 \\
\hline
\end{tabular}

* Dx = diagnosis. 
TABLE 2: Cross table of HGGs and METs: results of iMRI and $5-\mathrm{ALA}^{*}$

\begin{tabular}{lrrrrrrrr}
\hline & \multicolumn{3}{c}{ iMRI } & & \multicolumn{3}{c}{ 5-ALA } \\
\cline { 2 - 5 } \cline { 6 - 8 } Histo Dx of Sample & Neg & Int & Pos & & Neg & Int & Pos \\
\hline HGGs & 23 & 17 & 28 & & 6 & 4 & 58 \\
$\quad$ tumor & 23 & 5 & 13 & & 8 & 7 & 26 \\
$\quad$ infiltration zone & 23 & 1 & 1 & & 4 & 1 & 0 \\
$\quad$ no tumor & 3 & & & & & & \\
$\quad \begin{array}{l}\text { METs } \\
\quad \text { tumor }\end{array}$ & 1 & 1 & 6 & & 1 & 1 & 6 \\
$\quad$ infiltration zone & 1 & 0 & 0 & & 0 & 0 & 1 \\
$\quad$ no tumor & 2 & 1 & 1 & & 3 & 1 & 0 \\
\hline
\end{tabular}

* Shaded fields show the ideal distribution of a test. Histo = histopathological; int = intermediate; neg = negative; pos = positive.

ity of each technique; iMRI showed a sensitivity of 0.41 compared with 5-ALA, in which the calculated sensitivity for tumor detection was 0.85 . To compare sensitivity and specificity statistically, we assessed distribution of test results using the McNemar test. The distribution of results of iMRI and 5-ALA in the histological condition "tumor positive" (sensitivity) was significantly different between both imaging techniques ( $p<0.001)$, as opposed to specificity, which was higher in iMRI (0.70) than in 5-ALA (0.43). The distribution of test results in the condition "tumor negative" (specificity) was significantly different between iMRI and 5-ALA ( $\mathrm{p}<0.001)$.

We calculated ROC curves and AUC for both

TABLE 3: Calculations of performance measures for detection of HGGs and METs with iMRI and 5-ALA

\begin{tabular}{lccccc}
\hline \multirow{2}{*}{ Measure } & \multicolumn{2}{c}{ Solid Tumor } & & \multicolumn{2}{c}{ Pathological Tissue } \\
\cline { 2 - 3 } \cline { 5 - 6 } HGGs & iMRI & 5-ALA & & iMRI & $5-A L A$ \\
sensitivity & $0.41 \dagger$ & $0.85 \dagger$ & & $0.66 \dagger$ & $0.91 \dagger$ \\
specificity & $0.70 \dagger$ & $0.43 \dagger$ & & $0.60 \dagger$ & $0.80 \dagger$ \\
PPV & 0.67 & 0.69 & & 0.97 & 0.99 \\
NPV & 0.70 & 0.43 & & 0.06 & 0.22 \\
false-neg rate & 0.47 & 0.33 & & 0.47 & 0.33 \\
false-pos rate & 0.33 & 0.31 & & 0.33 & 0.31 \\
OR & 1.60 & 4.46 & & 2.36 & 38.00 \\
METs & & & & & \\
sensitivity & $0.75 \ddagger$ & $0.75 \ddagger$ & & $0.88 \ddagger$ & $0.88 \ddagger$ \\
specificity & $0.80 \ddagger$ & $0.80 \ddagger$ & & $0.50 \ddagger$ & $0.75 \ddagger$ \\
PPV & 0.86 & 0.86 & & 0.78 & 0.89 \\
NPV & 0.80 & 0.80 & & 0.50 & 0.75 \\
false-neg rate & 0.25 & 0.25 & & 0.25 & 0.25 \\
false-pos rate & 0.14 & 0.14 & & 0.14 & 0.14 \\
OR & 12.00 & 12.00 & & 3.50 & 12.00 \\
\hline
\end{tabular}

* Represents tumor and infiltration zone.

† $p<0.001$, McNemar test.

$\ddagger p<0.05$, McNemar test. techniques (Fig. 1 left). The AUC was 0.577 (95\% CI 0.46-0.69) for iMRI and 0.627 (95\% CI 0.513-0.741) for 5-ALA. A significant difference from $0.5\left(\mathrm{H}_{0}\right.$ : $\mathrm{AUC}=$ 0.5 is consistent with $0.5=$ no discrimination) was found for 5-ALA ( $\mathrm{p}<0.031)$. We performed the same calculation for detection of pathological tissue, which includes infiltration zone and tumor, because these areas are the ideal targets for a gross-total resection in noneloquent locations. Results are compared with the findings for solid tumor (Table 3).

Specificity is distinctly higher in 5-ALA (0.80) if the infiltration zone is included in positive findings, whereas it drops to 0.60 in iMRI. We see a significantly higher specificity now in 5-ALA than in iMRI $(p<0.001)$. Sensitivity is significantly higher $(\mathrm{p}<0.001)$ in 5-ALA $(0.91)$ versus iMRI (0.66). The PPV is close to 1 in both techniques. However, the NPV is lower in iMRI (0.06) than in 5-ALA (0.22). The OR to find infiltration zone or solid tumor in a positive imaging finding increases extremely in 5-ALA (OR 38), whereas in iMRI in comparison with tumor alone it is only slightly higher (OR 2.36).

The ROC curves for detection of pathological tissue are found in Fig. 1 right. The AUC was 0.935 (95\% CI $0.847-1.023)$ in 5-ALA and 0.780 (95\% CI 0.519-1.041) in iMRI. Both imaging techniques showed no significant difference from the no-discrimination line.

Correlations were tested using Spearman rho (Table 4). Results of both imaging techniques did not correlate in our study. The 5-ALA technique correlated with final diagnosis of specimen $(p<0.001)$, whereas iMRI showed only a tendency toward a significant correlation $(\mathrm{p}<0.07)$ in this regard. Results of 5-ALA correlated with Ki 67 index and presence of vascular proliferations. No further correlations were found for iMRI.

\section{Comparison of Histopathological Results: METs}

Distribution in the cross table for MET (Table 2) did not show a distinct difference as seen in HGG. Similar results are found in 5-ALA and iMRI for solid tumor detection (Table 3). Both techniques show high results for PPV and NPV and low false-negative and false-positive rates. The OR for solid tumor detection is 12 using both 5-ALA and iMRI. No significant difference was found using the McNemar test ( $p>0.05$ ). The ROC curves show an identical distribution in iMRI and 5-ALA (Fig. 2 left). The AUC was 0.800 (95\% CI 0.531-1.069) for both. A tendency toward a significant difference from 0.5 was seen $(\mathrm{p}<0.079)$.

The additional detection of infiltration zone showed a lower specificity in iMRI (0.5 vs 0.75$)$ than in 5-ALA (Table 3); however, the difference was not significant. The NPV and PPV were lower in iMRI too. This resulted in a lower OR of 3.5 for iMRI vs 12 for 5-ALA. The AUC was higher in 5-ALA $(0.917,95 \%$ CI $0.758-1.075)$ than in iMRI (0.708 95\% CI 0.392-1.024). The value for 5-ALA (0.75) had a significant difference from the one for iMRI $(0.5)(\mathrm{p}<0.021)$.

Both imaging techniques showed significant correlation ( $p<0.022$, Table 4$)$. A significant correlation of iMRI and 5-ALA was found for final diagnosis of specimen, but the correlation coefficient was slightly higher in 


\section{Histopathological assessment at border zone with 5-ALA and iMRI}
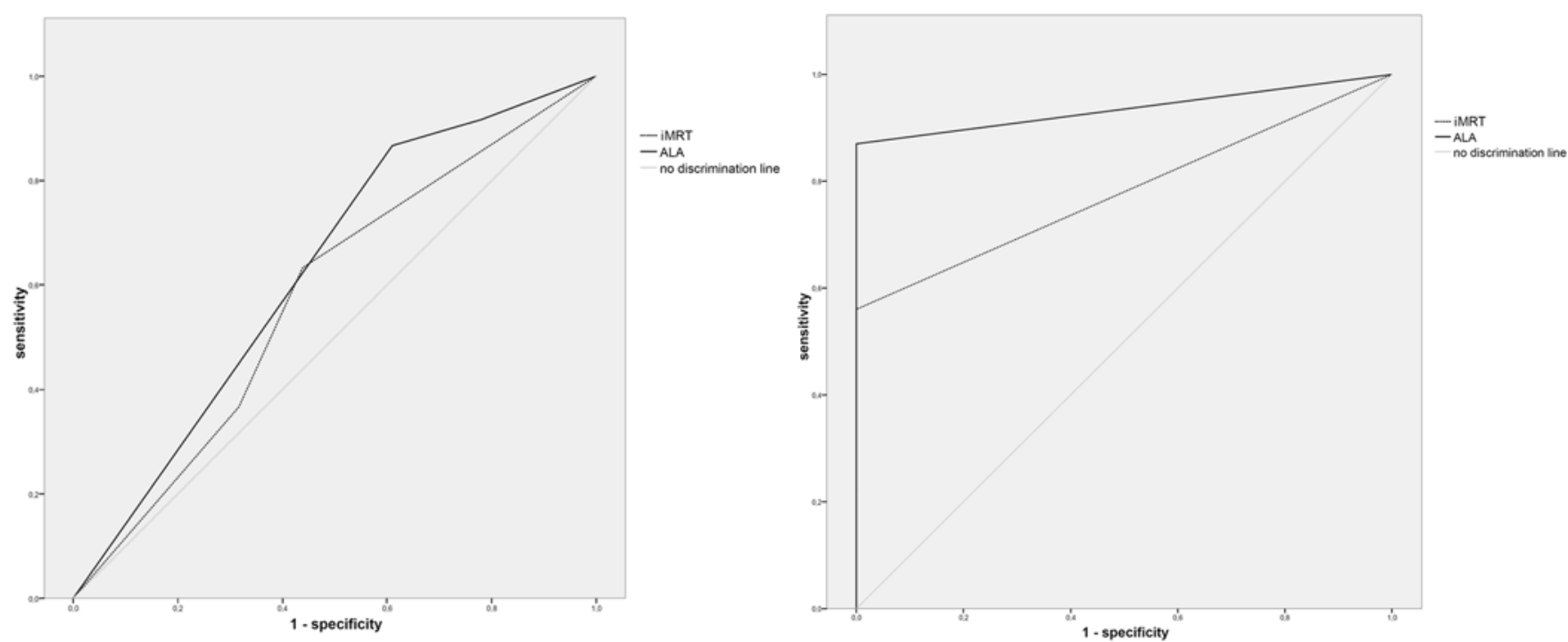

FIG. 1. Charts showing ROC curves for 5-ALA (solid line) and Gd-DTPA-enhanced iMRI (dotted line) for solid tumor detection (left) and for pathological tissue (right), including infiltration zone, in HGGs.

5-ALA than in iMRI. The Ki 67 index correlated highly with results of both imaging techniques. However, presence of vascular microproliferation correlated only with results of iMRI.

\section{Discussion}

The Gold Standard to Assess EOR in HGGs and METs is Postoperative Gd-DTPA-Enhanced MRI

All randomized controlled studies of HGG measure EOR based on residual postoperative contrast enhancement. ${ }^{5,11,12,14,16,20}$ However, histopathological assessment and PET/CT resection control studies have elucidated that active tumor tissue can be found beyond that area of Gd-DTPA uptake. Because contrast enhancement only depicts tumor areas with a disrupted blood-brain barrier, it might not show invasive areas at the border zone of lesions. ${ }^{1}$ The only tool other than iMRI established in clinical routine that shows residual tumor in situ is 5-ALAbased fluorescence. Preliminary data comparing 5-ALA fluorescence with preoperative MRI suggest a higher sensitivity for tumor tissue detection. ${ }^{19}$ However, due to brain shift, a histopathologically based comparison of 5-ALA and MRI has so far not been possible without significant bias. Therefore an exact comparison of Gd-DTPA enhancement and 5-ALA fluorescence, especially at the border zone of contrast-enhancing intracranial lesions, has to be performed based on iMRI. Data for the impact of 5-ALA as a resection control are rare, especially for

TABLE 4: Correlation of items calculated using the Spearman rho test in HGGs and METs*

\begin{tabular}{|c|c|c|c|c|c|c|}
\hline & 5-ALA & iMRI & Final Dx of Sample & Share of Necrosis & Ki 67 Index & VMPs \\
\hline \multicolumn{7}{|l|}{ HGGs } \\
\hline \multicolumn{7}{|l|}{ iMRI } \\
\hline corr coeff & 0.029 & 1 & 0.148 & 0.123 & 0.130 & 0.112 \\
\hline$p<$ & 0.388 & NA & 0.070 & 0.122 & 0.112 & 0.146 \\
\hline \multicolumn{7}{|l|}{ 5-ALA } \\
\hline corr coeff & 1 & 0.029 & $0.303 \dagger$ & 0.155 & $0.196 \ddagger$ & $0.296 \dagger$ \\
\hline$p<$ & NA & 0.388 & 0.001 & 0.070 & 0.032 & 0.002 \\
\hline \multicolumn{7}{|l|}{ METs } \\
\hline \multicolumn{7}{|l|}{ iMRI } \\
\hline corr coeff & $0.544 \ddagger$ & 1 & $0.514 \ddagger$ & 0.342 & $0.898 \dagger$ & $0.913 \ddagger$ \\
\hline$p<$ & 0.022 & NA & 0.036 & 0.227 & 0.008 & 0.015 \\
\hline \multicolumn{7}{|l|}{ 5-ALA } \\
\hline corr coeff & 1 & $0.544 \ddagger$ & $0.646 \dagger$ & 0.342 & $0.826 \ddagger$ & 0.304 \\
\hline$p<$ & NA & 0.022 & 0.009 & 0.227 & 0.021 & 0.309 \\
\hline
\end{tabular}

* Corr coeff = correlation coefficient; NA = not applicable; VMPs = vascular microproliferations.

$\dagger p<0.01$ single sided.

$\ddagger p<0.05$ single sided. 

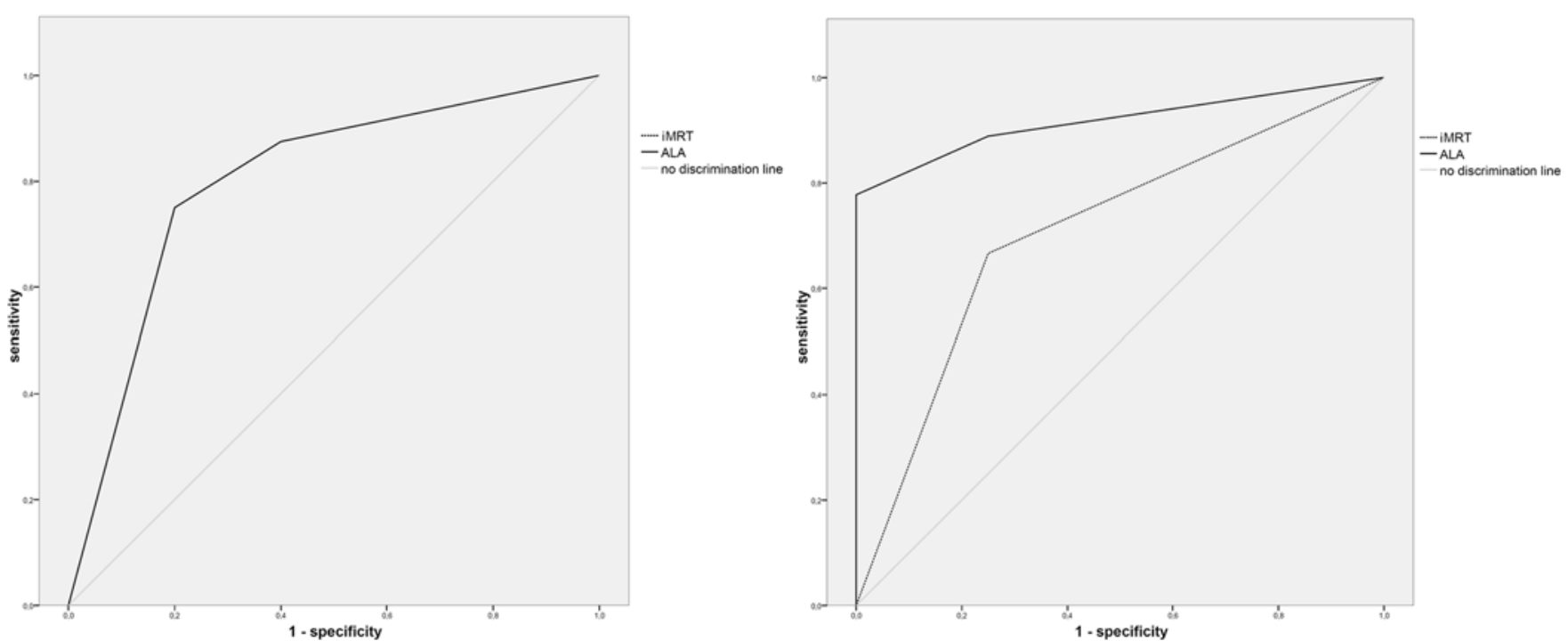

FIG. 2. Charts showing ROC curves for 5-ALA (solid line) and Gd-DTPA-enhanced iMRI (identical distribution in left panel, dotted line in right) for solid tumor detection (left) and for pathological tissue (right), including infiltration zone, in METs.

metastatic lesions. To our knowledge we provide the first histopathological evaluation of both techniques based on exact spatial intraoperative location.

\section{High-Grade Gliomas (WHO Grade IV GBMs)}

In our study iMRI and 5-ALA detected the targeted lesion in all patients with HGGs. Final histopathological assessment confirmed WHO Grade IV GBM in all HGGs in the series. Our study for the first time demonstrates a missing correlation between 5-ALA fluorescence and Gd-DPTA enhancement at the border zone of HGGs. So far, the opposite has been postulated in previous reports..$^{21,23-25}$ However, at the time of this writing a histopathology based assessment of iMRI and 5-ALA had not been published. Roberts et al. ${ }^{17}$ compared preoperative MRI and 5-ALA based on navigated biopsy samples. The authors found a significant correlation of contrast enhancement and 5-ALA fluorescence. Due to the comparison with preoperative MRI, a bias due to brain shift exists in that report. Additionally, only 11 patients were included.

In our study we show a significantly higher sensitivity for tumor detection at the border zone of HGG by using 5-ALA. The ROC curves demonstrate a superiority of 5-ALA to iMRI in this respect. Data from an actual meta-analysis by Zhao et al. ${ }^{33}$ of the existing 5-ALA studies show a similar sensitivity to that found in our study. Specificity for solid tumor detection is lower in our data in comparison with the published data of $0.89 \%$. However, the histopathological definition of "tumor" is used heterogeneously in the literature. Some authors include infiltration zone, which might generate a higher truenegative rate (specificity). In our study we distinguished between solid tumor, which fulfilled all WHO Grade IV GBM criteria, and "pathological tissue," which implies solid tumor and infiltrating tumor cells that do not meet all Grade IV GBM criteria. The specificity of 5-ALA for pathological tissue in our series was $80 \%$. The results for detection of pathological tissue, including ROC and AUC, are very similar to the results of the above-mentioned meta-analysis (AUC 0.93 vs 0.94).

Few histopathological studies exist in which the correlation of 5-ALA fluorescence and histopathological findings are assessed. At the time of this writing, to our knowledge no study has been published comparing histopathological findings of Gd-DTPA-enhanced iMRI and 5-ALA. The 5-ALA technique was shown to be a marker for anaplastic foci and increased tumor cell density. ${ }^{2,29}$ Our data confirm these previous findings for infiltrating tumor at the border zone of contrast-enhancing lesions. Invasive tumor areas were also found in 5-ALA-negative specimens. However, the rate was distinctly lower than in iMRI-negative samples. The detection rate obtained using the so far still experimental technique of intraoperative fluorescence spectroscopy might be higher than that found using the above-mentioned imaging devices. Yet the spectroscopy technique still needs to be validated in clinical routine. $6,10,23$

Our data suggest a higher detection rate for tumor and infiltration zone in 5-ALA as opposed to iMRI. In Gd-DTPA-enhanced iMRI a high number of tumor-positive areas are missed. On the other hand, the specificity for solid tumor detection of 5-ALA was significantly lower in comparison with iMRI. The 5-ALA technique shows a higher rate of false-positive results for infiltration zone. However, the infiltration zone has to be considered as part of the tumor although it does not fulfill all WHO criteria for a solid main tumor. Early tumor recurrence might arise from these residual parts. Thus most authors would agree that a "supramaximal" resection including infiltrating active tumor tissue is recommended if the lesion is in noneloquent areas.

We showed a significant advantage of 5-ALA, especially for detection of pathological tissue in general. Here sensitivity and specificity was significantly higher than in iMRI. In contrast to iMRI, 5-ALA correlated significantly with histopathological diagnosis and typical histopathological features of HGG such as Ki 67 index and 


\section{Histopathological assessment at border zone with 5-ALA and iMRI}

vascular proliferations. The OR for detection of pathological tissue increased up to 38 when using 5-ALA. The iMRI technique has a high specificity for tumor detection in HGG. However, our data show that surgeons relying on Gd-DTPA enhancement alone might miss important areas to resect at the border zone of lesions in glioma surgery. Thus, especially if a gross-total resection is intended in noneloquent areas, the use of 5-ALA in addition to iMRI is superior to iMRI alone from a histopathological perspective. This conclusion does not postulate a superiority of 5-ALA-guided resection alone to iMRI-guided resection alone. Our data only refer to a combined use of both techniques in a standardized study protocol. The sole use of 5-ALA has well-known disadvantages. Obscured tumor fluorescence, for example, might easily be missed without an additional iMRI scan.

\section{Metastatic Lesions}

Patients harboring a metastatic lesion showed visible 5-ALA fluorescence in $72 \%$ of cases. Fluorescence was independent of the etiology of primary lesions, as reported before by Kamp et al. ${ }^{8}$ Apart from that publication, data on 5-ALA fluorescence in cerebral metastatic lesions are rare.

In contrast to $\mathrm{HGG}, \mathrm{iMRI}$ and 5-ALA correlate significantly for depiction of residual tumor at the border zone of the lesion. Sensitivity and specificity are high in both techniques. However, as ROC curves and AUC suggest, for additional detection of the infiltration zone 5-ALA might have a slight benefit over iMRI alone.

Yet, patients without 5-ALA enhancement were excluded from the prospective study. Therefore, calculations of sensitivity and specificity do not contain these missing cases. Hence, for METs the additional benefit of 5-ALA-guided resection compared to iMRI-guided resection is debatable. In general, use of 5-ALA in METs has the disadvantage that fluorescence enhancement is not predictable and a significant share (27\% in our series) of lesions are fluorescence negative. An important step could be the identification of overexpression of cadherin 13 as a predictor of absent fluorescence in gliomas. ${ }^{27}$ Yet, so far no histological or clinical predictors for a positive protoporphyrin IX accumulation have been identified in METs. However, from the clinical perspective a lesion that is suspected of being a MET might turn out to be a GBM intraoperatively. Kamp et al. ${ }^{9}$ found a rate of $16 \%$ in their series, and they recommended "when in doubt, choose ALA." In our prospective series, test results in the METs subgroup are limited by small sample size. However, from our point of view exact tumor control is of high clinical benefit for a patient with a single brain MET and stable systemic disease. The prognosis for these patients often is much better compared with HGG. Thus, the additional benefit of 5-ALA as an adjunct to iMRI with the aim of a more precise detection of infiltration zone might be advantageous for some of these patients. Therefore further studies are needed to identify these cases.

Results of HGG are based on patients harboring WHO Grade IV GBM lesions alone, thus reflecting the majority of cases in clinical routine. However, data for sensitivity and specificity must not be generalized to WHO Grade III lesions.
Test results might be limited because negative control specimens were not harvested from normal brain tissue on a routine basis. Therefore the rate of false-negative results might be underestimated. However, results are comparable to those in the above-mentioned literature referring to 5-ALA. Additionally, this bias would affect both iMRI and 5-ALA and would not favor superior results of either technique.

Results of our study only reflect the border zone of contrast-enhancing lesions. Detection of solid tumor was not assessed because clinical relevance is minimal. In treating HGGs and METs the surgeon will usually locate and microsurgically remove the solid lesion, using white light resection and neuronavigation. The value of intraoperative imaging turns to account at the border zone of lesions.

As mentioned above, from our data no conclusion can be drawn whether 5-ALA alone is superior to iMRI alone. Our study protocol was set up to address the differences in detection of tumor tissue at the border zone of the resection cavity. We doubt the superiority of a solely 5-ALA-based resection to iMRI. In studies using 5-ALA, the issue of loss of accuracy of neuronavigation leading to an increase of neurological deficits in eloquent areas is described. Furthermore, tumor fluorescence is easily obscured by blood, spatula, or cottonoid, which is excluded using iMRI. ${ }^{25}$ Our results compare 5-ALA only with the standard protocol for Gd-DTPA-enhanced T1-weighted imaging. Further additional intraoperative MRI sequences were not evaluated. Intraoperative MRI spectroscopy and perfusion-weighted iMRI are promising techniques to detect tumor invasion beyond Gd-DTPA enhancement, according to the first clinical studies..$^{15,18}$

Our study shows a significant difference of tumor detection at the border zone of GBMs. Especially since detection of the infiltration zone is significantly superior in 5-ALA than in iMRI, we can expect a synergistic effect beneficial to the maximizing of EOR for application in the iMRI environment. Two studies with small sample sizes $(n=11$ and $n=8)$ have been published in which EOR was addressed using a combination of 5-ALA and iMRI. Both authors suggest a synergistic effect of 5-ALA and iMRI. However, due to these studies' small sample sizes no final conclusion can be drawn..$^{4,28}$

In our opinion the combined use of both techniques might increase safety of 5-ALA-guided resection close to eloquent areas and would overcome the issue of missed fluorescence in concealed regions of the resection cavity. Furthermore, the lower specificity of 5-ALA in comparison with iMRI would be balanced. We would therefore encourage establishing a prospective randomized trial to assess EOR and progression-free survival by comparing 5-ALA-based resection alone with the "combined imaging approach" in which iMRI and 5-ALA are used.

\section{Conclusions}

Imaging results of 5-ALA and iMRI are significantly different at the border zone of HGG. The 5-ALA technique has a significantly higher sensitivity and a lower specificity for tumor detection than Gd-DTPA-enhanced 


\section{J. Coburger et al.}

iMRI in HGG. For detection of pathological tissue at the border of the resection cavity, 5-ALA fluorescence is significantly superior to Gd-DTPA-enhanced iMRI. Thus, use of 5-ALA in addition to iMRI might be beneficial to maximize EOR in HGGs. Clinical synergistic effects need to be evaluated in a prospective randomized trial.

In metastatic lesions 5-ALA and iMRI yield equally good results. Yet, due to the high rate of nonfluorescent lesions our data show no additional benefit of 5-ALA to iMRI in METs. However, further studies are needed for a final conclusion.

\section{Acknowledgments}

We thank Mrs. Brigitta Grolik, Mrs. Christine Mayer, and Mr. Harry Watzel, who made the conduct of the study possible due to their outstanding work and extraordinary commitment in the daily clinical routine.

\section{Disclosure}

Dr. Thal is a consultant for GE Healthcare, and has collaborated on another study with Novartis Pharma. The authors report no conflict of interest concerning the materials or methods used in this study or the findings specified in this paper.

Author contributions to the study and manuscript preparation include the following. Conception and design: Coburger, Scheuerle, Thal, Hlavac, Wirtz, König. Acquisition of data: all authors. Analysis and interpretation of data: Coburger. Drafting the article: Coburger, Thal, Wirtz, König. Critically revising the article: Engelke, Scheuerle, Thal, Hlavac, Wirtz, König. Approved the final version of the manuscript on behalf of all authors: Coburger. Statistical analysis: Coburger. Administrative/technical/material support: Wirtz. Study supervision: Coburger, Wirtz, König.

\section{References}

1. Arbizu J, Tejada S, Marti-Climent JM, Diez-Valle R, Prieto E, Quincoces G, et al: Quantitative volumetric analysis of gliomas with sequential MRI and ${ }^{11} \mathrm{C}$-methionine PET assessment: patterns of integration in therapy planning. Eur J Nucl Med Mol Imaging 39:771-781, 2012

2. Arita H, Kinoshita M, Kagawa N, Fujimoto Y, Kishima H, Hashimoto N, et al: ${ }^{11} \mathrm{C}$-methionine uptake and intraoperative 5-aminolevulinic acid-induced fluorescence as separate index markers of cell density in glioma: a stereotactic image-histological analysis. Cancer 118:1619-1627, 2012

3. Chang EF, Clark A, Smith JS, Polley MY, Chang SM, Barbaro NM, et al: Functional mapping-guided resection of low-grade gliomas in eloquent areas of the brain: improvement of longterm survival. Clinical article. J Neurosurg 114:566-573, 2011

4. Eyüpoglu IY, Hore N, Savaskan NE, Grummich P, Roessler K, Buchfelder M, et al: Improving the extent of malignant glioma resection by dual intraoperative visualization approach. PLoS ONE 7:e44885, 2012

5. Hess KR: Extent of resection as a prognostic variable in the treatment of gliomas. J Neurooncol 42:227-231, 1999

6. Ishihara R, Katayama Y, Watanabe T, Yoshino A, Fukushima T, Sakatani K: Quantitative spectroscopic analysis of 5-aminolevulinic acid-induced protoporphyrin IX fluorescence intensity in diffusely infiltrating astrocytomas. Neurol Med Chir (Tokyo) 47:53-57, 2007

7. Kamp MA, Dibué M, Niemann L, Reichelt DC, Felsberg J, Steiger HJ, et al: Proof of principle: supramarginal resection of cerebral metastases in eloquent brain areas. Acta Neurochir (Wien) 154:1981-1986, 2012

8. Kamp MA, Grosser P, Felsberg J, Slotty PJ, Steiger HJ, Reif- enberger G, et al: 5-aminolevulinic acid (5-ALA)-induced fluorescence in intracerebral metastases: a retrospective study. Acta Neurochir (Wien) 154:223-228, 2012

9. Kamp MA, Santacroce A, Zella S, Reichelt DC, Felsberg J, Steiger HJ, et al: Is it a glioblastoma? In dubio pro 5-ALA! Acta Neurochir (Wien) 154:1269-1273, 2012

10. Kantelhardt SR, Diddens H, Leppert J, Rohde V, Hüttmann G, Giese A: Multiphoton excitation fluorescence microscopy of 5 -aminolevulinic acid induced fluorescence in experimental gliomas. Lasers Surg Med 40:273-281, 2008

11. Kreth FW, Berlis A, Spiropoulou V, Faist M, Scheremet R, Rossner R, et al: The role of tumor resection in the treatment of glioblastoma multiforme in adults. Cancer 86:2117-2123, 1999

12. Lawrence YR, Mishra MV, Werner-Wasik M, Andrews DW, Showalter TN, Glass J, et al: Improving prognosis of glioblastoma in the 21st century: who has benefited most? Cancer 118:4228-4234, 2012

13. Louis DN, Ohgaki H, Wiestler OD, Cavenee WK, Burger PC, Jouvet A, et al: The 2007 WHO classification of tumours of the central nervous system. Acta Neuropathol 114:97-109, 2007 (Erratum in Acta Neuropathol 114:547, 2007)

14. Oszvald Á, Güresir E, Setzer M, Vatter H, Senft C, Seifert V, et al: Glioblastoma therapy in the elderly and the importance of the extent of resection regardless of age. Clinical article. J Neurosurg 116:357-364, 2012

15. Pamir MN, Özduman K, Yıldız E, Sav A, Dinçer A: Intraoperative magnetic resonance spectroscopy for identification of residual tumor during low-grade glioma surgery. Clinical article. J Neurosurg 118:1191-1198, 2013

16. Pichlmeier U, Bink A, Schackert G, Stummer W: Resection and survival in glioblastoma multiforme: an RTOG recursive partitioning analysis of ALA study patients. Neuro Oncol 10:1025-1034, 2008

17. Roberts DW, Valdés PA, Harris BT, Fontaine KM, Hartov A, Fan X, et al: Coregistered fluorescence-enhanced tumor resection of malignant glioma: relationships between $\delta$-aminolevulinic acid-induced protoporphyrin IX fluorescence, magnetic resonance imaging enhancement, and neuropathological parameters. Clinical article. J Neurosurg 114:595-603, 2011

18. Roder C, Bender B, Ritz R, Honegger J, Feigl G, Naegele T, et al: Intraoperative visualization of residual tumor: the role of perfusion-weighted imaging in a high-field intraoperative magnetic resonance scanner. Neurosurgery 72 (2 Suppl Operative):ons151-ons158, 2012

19. Roessler K, Becherer A, Donat M, Cejna M, Zachenhofer I: Intraoperative tissue fluorescence using 5-aminolevolinic acid (5-ALA) is more sensitive than contrast MRI or amino acid positron emission tomography ((18)F-FET PET) in glioblastoma surgery. Neurol Res 34:314-317, 2012

20. Sanai N, Berger MS: Glioma extent of resection and its impact on patient outcome. Neurosurgery 62:753-766, 2008

21. Schucht P, Beck J, Abu-Isa J, Andereggen L, Murek M, Seidel $\mathrm{K}$, et al: Gross total resection rates in contemporary glioblastoma surgery: results of an institutional protocol combining 5-aminolevulinic acid intraoperative fluorescence imaging and brain mapping. Neurosurgery 71:927-936, 2012

22. Senft C, Bink A, Franz K, Vatter H, Gasser T, Seifert V: Intraoperative MRI guidance and extent of resection in glioma surgery: a randomised, controlled trial. Lancet Oncol 12:997-1003, 2011

23. Stummer W, Novotny A, Stepp H, Goetz C, Bise K, Reulen $\mathrm{HJ}$ : Fluorescence-guided resection of glioblastoma multiforme by using 5 -aminolevulinic acid-induced porphyrins: a prospective study in 52 consecutive patients. J Neurosurg 93:1003-1013, 2000

24. Stummer W, Pichlmeier U, Meinel T, Wiestler OD, Zanella F, Reulen HJ: Fluorescence-guided surgery with 5-aminolevulinic 


\section{Histopathological assessment at border zone with 5-ALA and iMRI}

acid for resection of malignant glioma: a randomised controlled multicentre phase III trial. Lancet Oncol 7:392-401, 2006

25. Stummer W, Tonn JC, Mehdorn HM, Nestler U, Franz K, Goetz $\mathrm{C}$, et al: Counterbalancing risks and gains from extended resections in malignant glioma surgery: a supplemental analysis from the randomized 5-aminolevulinic acid glioma resection study. Clinical article. J Neurosurg 114:613-623, 2011

26. Stupp R, Hegi ME, Mason WP, van den Bent MJ, Taphoorn MJ, Janzer RC, et al: Effects of radiotherapy with concomitant and adjuvant temozolomide versus radiotherapy alone on survival in glioblastoma in a randomised phase III study: 5-year analysis of the EORTC-NCIC trial. Lancet Oncol 10:459-466, 2009

27. Suzuki T, Wada S, Eguchi H, Adachi J, Mishima K, Matsutani $\mathrm{M}$, et al: Cadherin 13 overexpression as an important factor related to the absence of tumor fluorescence in 5-aminolevulinic acid-guided resection of glioma. Laboratory investigation. J Neurosurg 119:1331-1339, 2013

28. Tsugu A, Ishizaka H, Mizokami Y, Osada T, Baba T, Yoshiyama M, et al: Impact of the combination of 5-aminolevulinic acid-induced fluorescence with intraoperative magnetic resonance imaging-guided surgery for glioma. World Neurosurg 76:120-127, 2011

29. Widhalm G, Wolfsberger S, Minchev G, Woehrer A, Krssak M, Czech T, et al: 5-Aminolevulinic acid is a promising marker for detection of anaplastic foci in diffusely infiltrating gliomas with nonsignificant contrast enhancement. Cancer 116:1545-1552, 2010
30. Yamahara T, Numa Y, Oishi T, Kawaguchi T, Seno T, Asai A, et al: Morphological and flow cytometric analysis of cell infiltration in glioblastoma: a comparison of autopsy brain and neuroimaging. Brain Tumor Pathol 27:81-87, 2010

31. Yoo H, Kim YZ, Nam BH, Shin SH, Yang HS, Lee JS, et al: Reduced local recurrence of a single brain metastasis through microscopic total resection. Clinical article. J Neurosurg 110: 730-736, 2009

32. Yordanova YN, Moritz-Gasser S, Duffau H: Awake surgery for WHO Grade II gliomas within "noneloquent" areas in the left dominant hemisphere: toward a "supratotal" resection. Clinical article. J Neurosurg 115:232-239, 2011

33. Zhao S, Wu J, Wang C, Liu H, Dong X, Shi C, et al: Intraoperative fluorescence-guided resection of high-grade malignant gliomas using 5-aminolevulinic acid-induced porphyrins: a systematic review and meta-analysis of prospective studies. PLoS ONE 8:e63682, 2013

Manuscript submitted October 11, 2013.

Accepted November 21, 2013.

Please include this information when citing this paper: DOI: 10.3171/2013.11.FOCUS13463.

Address correspondence to: Jan Coburger, M.D., Ludwig Heilmeyerstr. 2, 89312 Günzburg, Germany. email: jan.coburger@uniulm.de. 